\title{
Botryomycosis of Neck in Immunocompetent Female- An Uncommon Presentation
}

\author{
Ram Chander, Soumya Agarwal, Mahima Agarwal, Taru Garg and Kiran Agarwal \\ Department of Dermatology and STD, Lady Hardinge Medical College and Associated hospitals, New Delhi, India
}

\begin{abstract}
Botryomycosis is a chronic, suppurative, granulomatous, bacterial infection of the skin and subcutaneous tissues. The most common causative organisms are Staphylococcus aureus, and Pseudomonas $s p$. We report an interesting case of a 54 year old immunocompetent female who presented with a verrucous lesion over the left side of her neck since last 4 years. The case is reported for the persistence of lesion and its unique lymphatic spread. Resolution was achieved with a prolonged course of antibiotics (amoxicillin-clavulanic acid, ciprofloxacin, and vancomycin) and surgical debridement.
\end{abstract}

\section{Introduction}

Botryomycosis is a chronic granulomatous reaction to bacterial infection, most commonly caused by Staphylococcus aureus, and Pseudomonas sp., which contains granules resembling the sulphur granules of actinomycosis. The various underlying predisposing factors described for botryomycosis include trauma, diabetes mellitus, lung diseases, corticosteroid therapy, and HIV infection.

It was first considered to be a fungal infection by Rivolta, thus the term botryomycosis (from Greek, botrys-bunch of grapes, mycosisfungal origin). Later, the bacterial etiology was discovered and other terms including actinophytosis, staphylococcal actinophytosis, bacterial pseudomycosis and granular bacteriosis were suggested $[1,2]$.

\section{Case Report}

A 54 year old female presented with few asymptomatic raised skin lesions over the left side of her neck since 4 years. The lesions started as a pea-sized dome shaped lesion behind the left auricle which gradually progressed over 3-4 years to become more hypertrophic and involve almost whole of the left side of neck. The lesion was often associated with purulent discharge on trauma, and the patient gave history of undergoing repeated aspiration of pus by a private practitioner. The patient also complained of malaise, generalised weakness, insomnia and loss of appetite from the past 3-4 years, however, there was no history of significant weight loss. The patient denied any past history of tuberculosis in self or family. On cutaneous examination, four, well defined, discrete, hyper pigmented, verrucous, and crusted plaques of size ranging from $2 \times 2 \mathrm{~cm}$ to $14 \times 7 \mathrm{~cm}$ were present over left side of the neck in a lymphocutaneous pattern. The plaques were surrounded by erythematous margins, and covered with a heaped up yellow-brown crust. The removal of crust revealed foul smelling greenish-yellow pus stained with blood along with granulation tissue (Figures 1 and 2). There was no underlying swelling or lymphadenopathy.

A differential diagnosis of cutaneous tuberculosis, actinomycosis, deep fungal infection, and botryomycosis was considered. On investigations, Ziehl - Neelsen staining of purulent material, mycobacterial culture and PCR (Polymerase Chain Reaction) for Mycobacterium tuberculosis were negative. $\mathrm{KOH}$ preparation of purulent material, fungal culture, and Grocott-Gomori silver stain of the tissue were also negative. On bacterial culture, the i solate grew as a golden-yellow pigmented, opaque colony that was later diagnosed as S. aureus by Gram staining, and catalase and coagulase tests. Skin

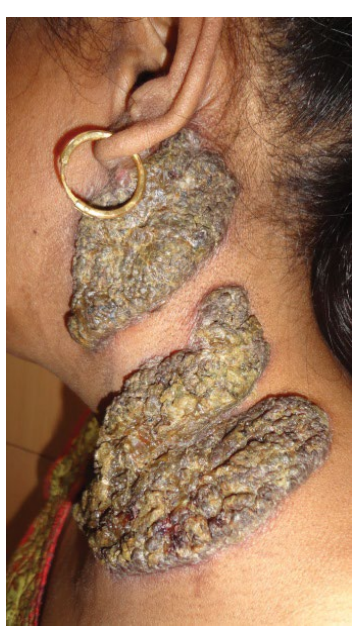

Figure 1: The removal of crust revealed foul smelling greenish-yellow pus stained with blood along with granulation tissue.

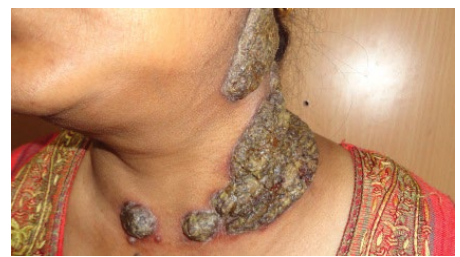

Figure 2: No underlying swelling or lymphadenopathy

*Corresponding author: Ram Chander, Department of Dermatology and STD Lady Hardinge Medical College and Associated hospitals, New Delhi, India, Tel: +91-8588971173; E-mail: chanderram41@gmail.com

Received February 04, 2016; Accepted February 27, 2016; Published March 05 2016

Citation: Chander R, Agarwal S, Agarwal M, Garg T, Agarwal K (2016) Botryomycosis of Neck in Immunocompetent Female- An Uncommon Presentation. J Microb Biochem Technol 8: 120-122. doi: 10.4172/1948-5948.1000273

Copyright: @ 2016 Chander R, et al. This is an open-access article distributed under the terms of the Creative Commons Attribution License, which permits unrestricted use, distribution, and reproduction in any medium, provided the original author and source are credited. 
Citation: Chander R, Agarwal S, Agarwal M, Garg T, Agarwal K (2016) Botryomycosis of Neck in Immunocompetent Female- An Uncommon Presentation. J Microb Biochem Technol 8: 120-122. doi: 10.4172/1948-5948.1000273

biopsy revealed colonies of Gram positive bacteria (Figures 3 and 4) and Splendore-Hoeppli phenomenon with chronic inflammatory infiltrate in the dermis (Figure 5). Her thyroid function tests suggested hypothyroidism, and levothyroxine $25 \mu \mathrm{g}$ was started for the same. Serum vitamin D3 level was very low $(7.8 \mathrm{ng} / \mathrm{ml})$, and was adequately supplemented. Other haematological and biochemical investigations, chest X-ray, USG abdomen, and HIV test were within normal limits, except for raised ESR (Erythrocyte Sedimentation Rate). Psychiatric evaluation revealed mood disorder, which was treated with escitalopram. The diagnosis of botryomycosis was confirmed and a prolonged course of amoxicillin-clavulanic acid, ciprofloxacin, and vancomycin antibiotics were used for treatment, along with surgical debridement.

\section{Discussion}

Botryomycosis is a rare, chronic, granulomatous, suppurative bacterial infection involving the skin and organs, where granules composed of bacterial masses resembling sulfur granules are formed. Cutaneous botryomycosis is a rare disease, scarcely described in the international literature [3]. The most frequent etiological agent is Staphylococcus aureus (40\%), followed by Pseudomonas sp (20\%) [2]. Other microorganisms reported are Escherichia coli, Proteus vulgaris, Bacillus spp, Actinobacillus lignieresii, etc. [1,3]. The inflammatory reaction is characterized by the presence of granules with an

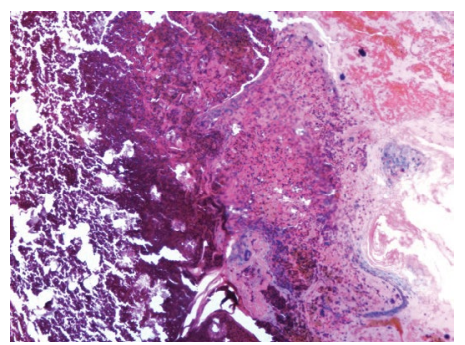

Figure 3: Skin biopsy revealed colonies of Gram positive bacteria

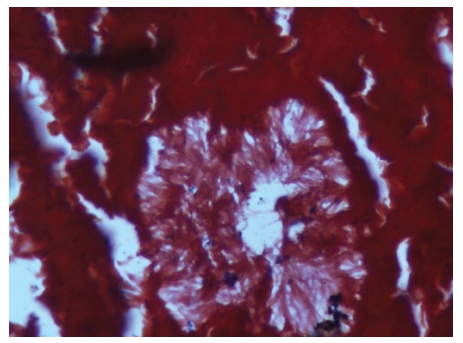

Figure 4: Skin biopsy revealed colonies of Splendore-Hoeppli.

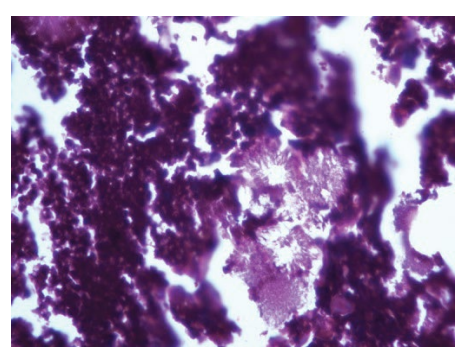

Figure 5: Splendore-Hoeppli phenomenon with chronic inflammatory infiltrate in the dermis. eosinophilic periphery corresponding to the immune response of the host. However, botryomycosis granules do not stain with GrocottGomori silver stain, as opposed to actinomycotic granules [2].

The skin lesions are pleomorphic, and may resemble cysts, abscesses, fistulas, nodules, plaques or ulcers. They are usually located on areas exposed to trauma (head, arms, legs) and genital regions $[1,3]$. Muscles, aponeuroses, tendons, bones and oral cavity can also be affected.

Cutaneous botryomycosis almost never develops in healthy individuals, with very few exceptions. Most of the reported cases were associated with conditions that attenuate the host's immune response, most commonly with HIV infection and AIDS. Our patient was reportedly HIV negative, and laboratory values did not point at a major immune deficiency. The crucial factors in the pathogenesis of botryomycosis remain unclear. An alteration in humoral immune response and specific cellular immunity has been postulated in its causation.

A history of injury is common in skin cases, and the importance of a foreign body as well as infection has been stressed [4,5]. The size of bacterial inoculation may be crucial and a delicate balance between host and pathogen has been regarded as significant.6 The predisposing factors associated to the disease are skin trauma, complicated postoperative period, diabetes mellitus, corticosteroid therapy in high doses or for a prolonged period, alcohol abuse, cystic fibrosis, and lung diseases [6,7]. Less often, malnutrition, glomerulonephritis, AIDS, asthma, Job syndrome and follicular mucinosis are associated with this condition $[2,7,8]$

The treatment of botryomycosis depends on identification of the etiological agent by means of direct examination, special stains and culture of secretion of the lesion. Antibiotic therapy is often used for prolonged period and, in some cases, surgical excision or drainage of lesions are required $[2,6]$.

Our case is interesting because of its persistence, site, and unique lymphatic spread. Botryomycosis is extremely rare in the head and neck, and consideration of this entity in the differential diagnosis is critical to the diagnosis. Secondly, lesion similar to tuberculosis in a vitamin D deficient lady with mood disorder and hypothyroidism was worth reporting. However, it was difficult to establish the chronology of events in our case, as to whether hypothyroidism led to mood disorder and thereby ignorance of the lesion, or abnormal tissue reaction in the setting of severe vitamin D deficiency and aberrant host immune response incited the persistence of lesion. The initiation of vitamin D supplementation, levothyroxine along with antidepressants led to an overall improvement in the patient's compliance and wellbeing which aided the resolution of lesions to prolonged course of antimicrobials comprising amoxicillin-clavulanic acid, ciprofloxacin, and vancomycin.

\section{References}

1. Mehregan DA, Su WP, Anhalt JP (1991) Cutaneous botryomycosis. J Am Acad Dermatol 24: 393-396.

2. Bonifaz A, Carrasco E (1996) Botryomycosis. Int J Dermatol 35: 381-388.

3. Fernandes NC, Maceira JP, Knackfuss IG, Fernandes N (2002) Botriomicose cutânea. An Bras Dermatol 77: 65-70.

4. Yencha MW, Walker CW, Karakla DW, Simko EJ (2001) Cutaneous botryomycosis of the cervicofacial region. Head Neck 23: 594-598.

5. Hacker P (1983) Botryomycosis. Int J Dermatol 22: 455-458. 
Citation: Chander R, Agarwal S, Agarwal M, Garg T, Agarwal K (2016) Botryomycosis of Neck in Immunocompetent Female- An Uncommon Presentation. J Microb Biochem Technol 8: 120-122. doi: 10.4172/1948-5948.1000273

6. Brunken RC, Lichon-Chao N, van der Broek H (1983) Immunologic abnormalities in botryomycosis. A case report with review of the literature. $J$ Am Acad Dermatol 9: 428-434.

7. de Vries HJ, van Noesel CJ, Hoekzema R, Hulsebosch HJ (2003) Botryomycosis in an HIV-positive subject. J Eur Acad Dermatol Venereol 17: 87-90.

8. Harman RR, English MP, Halford M, Saihan EM, Greenham LW (1980) Botryomycosis: a complication of extensive follicular mucinosis. Br J Dermato 102: 215-222. 